\title{
A Living Laboratory for the Design and Evaluation of Ubiquitous Computing Technologies
}

\author{
Stephen S. Intille, Kent Larson, J.S. Beaudin, J. Nawyn, E. Munguia Tapia, P. Kaushik \\ House_n, Massachusetts Institute of Technology \\ Cambridge, MA 02139 USA \\ intille@mit.edu
}

\begin{abstract}
We introduce the PlaceLab, a new "living laboratory" for the study of ubiquitous technologies in home settings. The PlaceLab is a tool for researchers developing context-aware and ubiquitous interaction technologies. It complements more traditional data gathering instruments and methods, such as home ethnography and laboratory studies. We describe the data collection capabilities of the laboratory and current examples of its use.
\end{abstract}

\section{Author Keywords}

Ubiquitous computing, context-aware, living laboratory, home, sensors, research methods, ethnography.

\section{ACM Classification Keywords}

H5.m. Information interfaces and presentation (e.g., HCI): Miscellaneous.

\section{INTRODUCTION}

Mobile and ubiquitous computing devices are transforming the way that people interact with digital information. Extended sessions at desktop computers are giving way to rapid interactions with technology embedded in everyday life. Ideally, emerging human-computer interfaces will be able to automatically detect context and present and gather information without unduly disrupting the complex activity inherent to any real and dynamic environment such as the home [3].

Studying behavior in naturalistic living environments allows researchers to better understand how to create technologies that respond to and respect the complexity of life. As a complement to existing tools and methodologies for gathering data on behavior and use of technology in home settings (e.g., laboratory user studies, surveys, interviews, ethnographic observation) we have developed a

Copyright is held by the author/owner(s).

CHI 2005, April 2-7, 2004, Portland, Oregon, USA.

ACM 1-59593-002-7/05/0004. live-in, apartment-scale research facility called the PlaceLab

The PlaceLab is a real home where the routine activities and interactions of everyday home life can be observed, recorded for later analysis, and experimentally manipulated. Volunteer research participants individually live in the PlaceLab for days or weeks, treating it as a temporary home. Meanwhile, a detailed description of their activities is recorded by sensing devices integrated into the fabric of the architecture.

\section{THE PLACELAB}

Figure 1 shows interior photos of the PlaceLab facility, which is located in a residential condominium building within a Cambridge, MA neighborhood. The 1000 square foot lab consists of a living room, dining area, kitchen, small office, bedroom, full bath and half bath, as shown in the floor plan in Figure 2. The PlaceLab is optimized for studies that would benefit from multi-day or multi-week observation of single individuals living alone. In 2003, 26\% of U.S. households consisted of a person living alone [8].

The interior of the PlaceLab is formed by 15 prefabricated and reconfigurable cabinetry components. Each contains a micro controller, an addressable speaker system, and a network of 25 to 30 sensors. New sensors can be easily added to this network as required. Existing sensors record a complete audio-visual record of activity. All sensing devices are discreetly integrated into the cabinetry, appliances, and furnishings and fixtures.

Eighty small, wired switches detect on-off and open-closed events, such as the opening of the refrigerator, the shutting of the linen closet, or the lighting of a stovetop burner. Interior conditions of the apartment are captured using distributed temperature (34), humidity (10), light (5), and barometric pressure (1) sensors. The PlaceLab also features electrical current sensors (37), water flow (11) and gas flow (2) sensors. Small wireless sensors that detect movement can be easily taped onto any movable objects or worn by the participant with wrist bands or ankle bands [9]. Nine infrared cameras, 9 color cameras, and 18 microphones are distributed throughout the apartment in cabinet components and above working surfaces, such as the office desk and kitchen counters. Twenty computers use image-processing algorithms to select the 4 video streams and 1 audio stream 

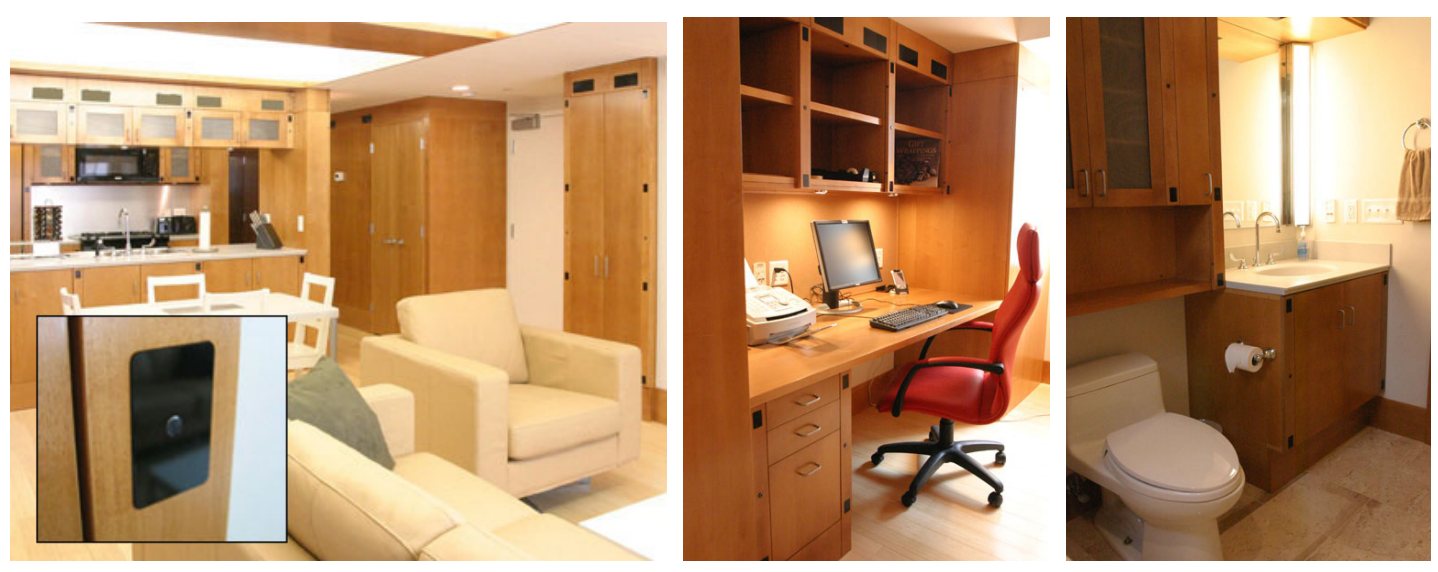

Figure 1: The PlaceLab living room and kitchen, office, and master bath. All of the observational sensing is built directly into the cabinetry. Although the sensors are ubiquitous, they become part of the design aesthetic (small black windows). Pilot volunteers have expressed that they are easy to forget. The inset in the left image shows a microphone.

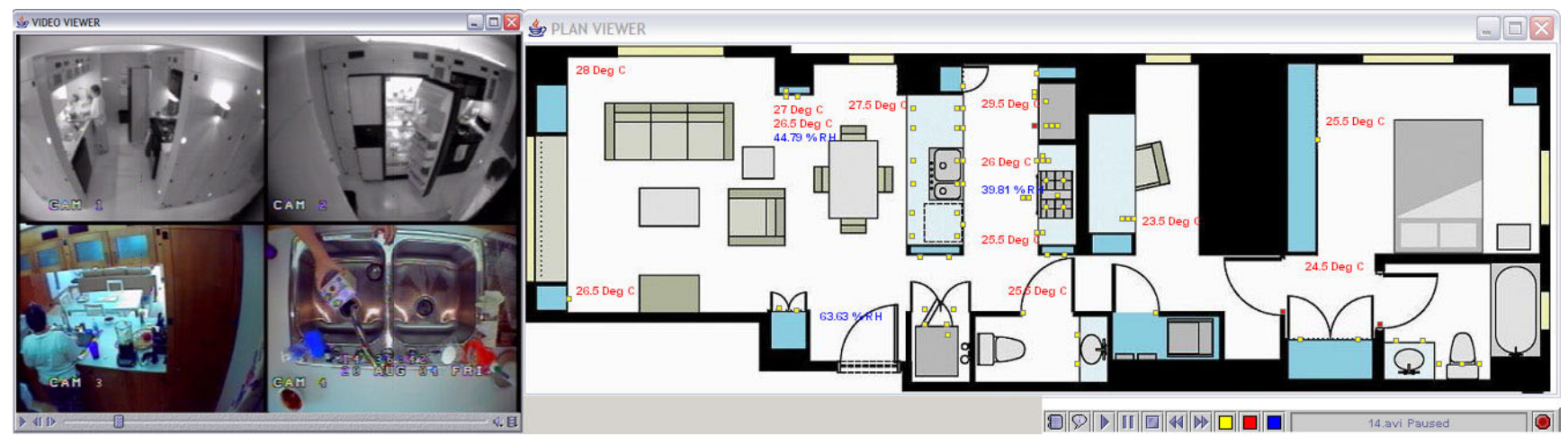

Figure 2: Annotation software permits the researcher to study data collected from the PlaceLab by watching 4 streams of automatically-selected "good" video views, listening to one automatically selected audio stream, and displaying sensor activations on a floor plan of the environment. Here the floor plan shows all switch sensors currently installed in the lab (small dots) and temperature sensor output. The researcher can use particular sensor activations to reduce search and annotation time.

that may best capture an occupant's behavior, based on motion and the camera layout in the environment. These data streams are synchronized with all other sensor data and saved to disk.

PlaceLab data streams can be used to develop and test new context-detection algorithms and to prototype contextaware computing applications for desktop and mobile devices.

PlaceLab data recordings can also be loaded into a custom visualization tool that permits the user to click on any sensor in the environment and immediately be taken to an audio-visual record of what was happening at the time of the sensor activation (see Figure 2). This may dramatically reduce search and observation time when researchers and designers wish to focus on particular types of behaviors.

\section{COMPLEMENTING EXISTING TOOLS AND METHODS}

A key motivation for the creation of the PlaceLab arose from our prior work developing context-detection algorithms in traditional laboratory settings. Controlled laboratory studies allowed dense sensor installation useful for the study of behavior and development of new contextaware algorithms, but simulated rooms or short stays severely constrained behavior variability.

As an alternative approach, we have also installed portable sensors in real homes, but practical limitations dictated that only a subset of a laboratory system could be deployed at once.

A living laboratory such as the PlaceLab is a compromise option that can be used to help migrate work from the laboratory setting to the home. Although asking a person to move into any environment other than his or her own home will alter some behavior, a live-in lab may allow for more natural behavioral observation and data collection on everyday activities such as cooking, socializing, sleeping, cleaning, working from home, and relaxing than can be obtained from short laboratory visits. 


\begin{tabular}{|c|c|c|c|}
\hline & Some Benefits & Some Limitations & How PlaceLab May Complement \\
\hline $\begin{array}{l}\text { Surveys and } \\
\text { Interviews }\end{array}$ & $\begin{array}{l}\text { Large- } n \text { studies possible; can produce } \\
\text { data that can be statistically analyzed and } \\
\text { compared; can explore user's subjective } \\
\text { impressions: goals, intentions, histories, } \\
\text { and perceptions }\end{array}$ & $\begin{array}{l}\text { Subject to recall and selective } \\
\text { reporting biases; poor at capturing } \\
\text { chains of causality }\end{array}$ & $\begin{array}{l}\text { Enable survey validation, including elucidation } \\
\text { of respondent errors; provide examples of } \\
\text { specific behavior for interview subjects to } \\
\text { discuss }\end{array}$ \\
\hline $\begin{array}{l}\text { Experience } \\
\text { Sampling (e.g., } \\
[1])\end{array}$ & $\begin{array}{l}\text { Less reliant on recall than } \\
\text { survey/interviews; can produce data that } \\
\text { can be statistically analyzed and } \\
\text { compared; possible to capture causality } \\
\text { and influence of context }\end{array}$ & $\begin{array}{l}\text { Disruptive to subject activities; } \\
\text { burdens participants; coarse-grain } \\
\text { sampling; no independent observer }\end{array}$ & $\begin{array}{l}\text { Can help validate new sampling studies by } \\
\text { comparing observed with reported events; } \\
\text { permits sensor-triggered interruption timing to } \\
\text { improve data quality and reduce subject burden }\end{array}$ \\
\hline $\begin{array}{l}\text { Direct } \\
\text { Observation }\end{array}$ & $\begin{array}{l}\text { Can provide rich descriptions of behavior } \\
\text { in authentic contexts }\end{array}$ & $\begin{array}{l}\text { Costly, time-consuming; invasive; } \\
\text { observer introduces bias }\end{array}$ & $\begin{array}{l}\text { Create library of examples studied by multiple } \\
\text { observers; sensor-activations can act as indices } \\
\text { for quick identification of behaviors of interest }\end{array}$ \\
\hline Portable Kits ([2]) & $\begin{array}{l}\text { Can provide descriptions of behavior in } \\
\text { authentic contexts; long-term observation } \\
\text { possible }\end{array}$ & $\begin{array}{l}\text { Density of sensing devices limited; } \\
\text { potentially invasive; limited sensor } \\
\text { coverage may require "interesting" } \\
\text { events be identified in advance. }\end{array}$ & $\begin{array}{l}\text { Pilot studies may help identify minimal sensor } \\
\text { requirements for research using portable kits; } \\
\text { can be used to compare behaviors in familiar vs. } \\
\text { new home environment }\end{array}$ \\
\hline $\begin{array}{l}\text { Demo Labs (e.g., } \\
[4,5])\end{array}$ & $\begin{array}{l}\text { Can help elicit feedback for new } \\
\text { solutions; allow rapid user testing }\end{array}$ & $\begin{array}{l}\text { Make assumptions about lifestyles; do } \\
\text { not typically capture existing behaviors } \\
\text { or extended interaction }\end{array}$ & $\begin{array}{l}\text { Provide authentic scenarios of everyday routines; } \\
\text { help generate ideas for how traditional labs can } \\
\text { be made more naturalistic }\end{array}$ \\
\hline $\begin{array}{l}\text { Short Tests in } \\
\text { Parts of Living } \\
\text { Labs [6]; Tests } \\
\text { With Limited } \\
\text { Sensors [7] }\end{array}$ & $\begin{array}{l}\text { Can provide descriptions of behavior in } \\
\text { restricted contexts; researcher can } \\
\text { experimentally manipulate environment } \\
\text { and interact with participants }\end{array}$ & $\begin{array}{l}\text { Impose significant constrains on task } \\
\text { execution, including ordering, multi- } \\
\text { tasking, use of objects, and type of } \\
\text { activity; may not capture all types of } \\
\text { activities or may be difficult to search } \\
\text { data for events of interest }\end{array}$ & $\begin{array}{l}\text { Permits long-term study of multi-tasking, task- } \\
\text { switching, and other activities that take place } \\
\text { throughout the home environment, not only in a } \\
\text { single part; captures a record of all activity with } \\
\text { real-time view selection; easy searching using } \\
\text { multi-modal sensor data to index events }\end{array}$ \\
\hline
\end{tabular}

Table 1: The identification of design criteria and the evaluation of human-computer interfaces for ubiquitous technologies is presently accomplished using multiple ethnographic and lab-based methodologies. Several of these are listed here, along with some of their significant benefits and limitations. The final column provides examples of how the PlaceLab may be employed to complement these methods.

In some sense, the PlaceLab is the "ubiquitous" analogy of the standard software usability lab. These labs remove people from the context of their actual workplace, but despite this simplification, the widespread use of the labs by commercial developers is evidence of their value. The PlaceLab, analogously, removes people from their actual homes, but in many cases it may still provide high quality data of value to ubiquitous computing researchers and developers.

As a living laboratory where human interaction with space, objects, technologies, and other people can be observed and experimentally manipulated, the PlaceLab has been designed to complement existing tools and methodologies being deployed for ubiquitous design. Table 1 delineates some of the benefits and limitations of existing methodologies and considers how the PlaceLab may be used in combination.

\section{PRELIMINARY AND EXAMPLE FUTURE STUDIES}

The PlaceLab opened in July 2004. Participants for three pilot PlaceLab stays of 10 days each were recruited via electronic mailing lists, posters, and word-of-mouth. Participant 1 was a man in his mid fifties who was working at home. The second participant was a semi-retired woman in her late fifties. The third participant was a woman in her mid-fifties who worked part-time. None were members of our research team. Each volunteer was compensated $\$ 250$ for participation.

Participants were asked to take part in three simultaneous study protocols that exploit observational capabilities of the living laboratory:

1. Activity recognition protocol: an empirical study on how simple sensors could be used for recognition of activities. Participants were asked to engage in their normal home routines at the PlaceLab while wearing three lightweight and wireless body limb acceleration sensors.

2. Activity recall protocol: an exploratory study of the differences between directly observable behaviors and the participants' recall of their behavior. During the course of their stay, participants were asked to complete five telephone interviews.

3. Dietary report protocol: an investigation of measurement error inherent to dietary surveys commonly used by medical researchers. ${ }^{1}$ Throughout

\footnotetext{
1 This study was designed and directed by Carla Kuesten and Roger Edwards of TIAX, LLC.
} 
the study period, participants were asked to complete dietary surveys over the telephone and on paper.

Each participant moved into the PlaceLab and was directed to conduct his or her life as normally as possible for the study period. Each stay yielded 200-250GB of data. Researchers responsible for each of the study protocols are currently reviewing the sensor data to identify and mark behaviors relevant to their research. An activity ontology is being developed so that each database acquired in the PlaceLab can be reused over time for purposes beyond the original intent. One such use, creating a library of everyday activity for designers, is discussed below.

\section{A LIBRARY OF EVERYDAY ACTIVITY}

The compilation of a library of video, audio, and sensor data on common activities in the home setting may, in itself, justify the efforts that are required to build and run a living lab. Like speech recognition algorithms, contextdetection algorithms may require a large corpus of training data [10]. However, even those who are developing concepts for new ubiquitous computing applications or studying existing home behavior and technology may find the PlaceLab datasets to be of value.

As most HCI developers know, a handful of concrete examples of real activity observation is worth hours of hypothetical discussions about what people may or may not do. Moreover, unusual examples can sometimes challenge the designer to break out of stereotypical assumptions, thereby leading to creative solutions.

Ubiquitous computing designers need common points of reference around which they can discuss ideas and develop new interfaces. What does searching behavior look like? How do people actually use their kitchen appliances? What do people do when getting ready for work? How much time do people spend waiting for something to happen? To date, PlaceLab studies have produced many examples of such events, and it is expected that continued research will produce a growing activity library with multi-modal sensor activation data and a complete audio-visual record.

\section{CONCLUSION}

Living laboratories such as the PlaceLab provide another tool for technologists, ethnographers, and others interested in studying and developing technologies that respond to home behaviors. Like usability labs, facilities like the PlaceLab are a compromise - allowing detailed data collection at the acknowledged cost of loss of some contextual complexity. Our three "pioneering" volunteers found the PlaceLab to be a pleasant environment, despite the intensive network of sensors. All three enthusiastically expressed a willingness to participate again. Based on these first three volunteers, we are documenting issues that must be considered when building and operating a living laboratory (e.g., how do people react to the sensors, how long does it take someone to acclimate).
The technical and administrative complexity of building and operating this kind of residential living laboratory is great, and we therefore expect the number of living laboratories available to HCI researchers to be small. However, our pilot testing has already created datasets that we could not have obtained in any other way. These datasets are a complete record of 10 days of home behavior of three individuals, with sensor data that simplifies the annotation of and searching for items of interest. Researchers who may be interested in using the PlaceLab or the PlaceLab datasets for HCI research are encouraged to contact the authors.

\section{REFERENCES}

[1] A. A. Stone and S. Shiffman, "Ecological momentary assessment (EMA) in behavioral medicine," Annals of Behavioral Medicine, vol. 16, pp. 199-202, 1994.

[2] S. S. Intille, E. Munguia Tapia, J. Rondoni, J. Beaudin, C. Kukla, S. Agarwal, L. Bao, and K. Larson, "Tools for studying behavior and technology in natural settings," in Proceedings of UbiComp 2003: Ubiquitous Computing. Berlin Heidelberg: Springer, 2003, pp. 157-174.

[3] G. D. Abowd and E. D. Mynatt, "Charting past, present, and future research in ubiquitous computing," $A C M$ Transactions on Computer-Human Interaction, vol. 7, pp. 29-58, 2000.

[4] B. de Ruyter and E. Aarts, "Ambient intelligence: visualizing the future," in Proceedings of the Working Conference on Advanced Visual Interfaces: ACM Press, 2004, pp. 203-208.

[5] "Living Tomorrow." http://www.livingtomorrow.com

[6] C. D. Kidd, R. J. Orr, G. D. Abowd, C. G. Atkeson, I. A. Essa, B. MacIntyre, E. Mynatt, T. E. Starner, and W. Newstetter, "The Aware Home: A living laboratory for ubiquitous computing research," in Proceedings of the Second International Workshop on Cooperative Buildings - CoBuild'99, 1999.

[7] K. Matsouoka, "Smart house understanding human behaviors: Who did what, where, and when," Proceedings of the 8th World Multi-Conference on Systems, Cybernetics, and Informatics, vol. 3, pp. 181185, 2004.

[8] J. Fields, "America's Families and Living Arrangements: 2003," U.S. Census Bureau, Washington, DC, Current Population Reports, P20-553 November 2004.

[9] E. M. Tapia, N. Marmasse, S. S. Intille, and K. Larson, "MITes: Wireless portable sensors for studying behavior," in Proceedings of Extended Abstracts Ubicomp 2004: Ubiquitous Computing, 2004.

[10]S. S. Intille, L. Bao, E. Munguia Tapia, and J. Rondoni, "Acquiring in situ training data for context-aware ubiquitous computing applications," in Proceedings of CHI 2004 Connect: Conference on Human Factors in Computing Systems. New York, NY: ACM Press, 2004, pp. 1-9. 